\title{
ON CONSTRUCTION OF A GLOBAL NUMERICAL SOLUTION FOR A SEMILINEAR SINGULARLY-PERTURBED REACTION DIFFUSION BOUNDARY VALUE PROBLEM
}

\author{
SAMIR KARASULJIĆ AND HIDAJETA LJEVAKOVIĆ
}

\begin{abstract}
A class of different schemes for finding the numerical solution of semilinear singularly-perturbed reaction-diffusion boundary-value problems was constructed. The stability of the difference schemes was proved, and the existence and uniqueness of a numerical solution were shown. After that, the uniform convergence with respect to a perturbation parameter $\varepsilon$ on a modified Shishkin mesh of order 2 has been proven. For such a discrete solution, a global solution based on a linear spline was constructed, also the error of this solution is in expected boundaries. Numerical experiments at the end of the paper, confirm the theoretical results. The global solutions based on a natural cubic spline, and the experiments with Liseikin, Shishkin and modified Bakhvalov meshes are included in the numerical experiments as well.
\end{abstract}

\section{INTRODUCTION}

We consider the semilinear boundary-value singularly-perturbed problem

$$
\varepsilon^{2} y^{\prime \prime}-f(x, y)=0, x \in(0,1), y(0)=y(1)=0,
$$

with the condition

$$
\frac{\partial f(x, y)}{\partial y}:=f_{y} \geqslant m>0,
$$

where $\varepsilon$ is a small positive perturbation parameter, $m$ is a positive constant, $y$ is a real-valued function of $x \in[0,1]$, and $f$ is a nonlinear function, $f(x, y) \in$ $C^{k}([0,1] \times \mathbb{R}), k \geqslant 2$. The problem $(1.1 \mathrm{a})$ under the condition $\left.1.1 \mathrm{~b}\right)$ has a unique solution, (see Lorenz [18]). It's a well-known fact in theory that the exact solution to 1.1a - 1.1b has two exponential boundary layers, i.e. near the end points $x=0$ and $x=1$.

Differential equations like $1.1 \mathrm{a}$ and similar occur in mathematical modeling of many problems in physics, chemistry, biology, engineering sciences, economics and even social sciences. Numerical solutions of singularly-perturbed boundary-value problems obtained by some classical methods are usually useless. That is because

2010 Mathematics Subject Classification. Primary: 65L11 Secondary: 65L20, 65L50.

Key words and phrases. Singular perturbation, Nonlinear, Boundary layer, Shishkin type mesh, Layer-adapted mesh, Uniform convergence. 
the exact solutions of the singularly-perturbed boundary-value problems depend on the perturbation parameter $\varepsilon$, but classical methods don't take into account the influence of the perturbation parameter. The singularly-perturbed problems require specially developed numerical methods in order to obtain the accuracy, which is uniform with respect to the parameter $\varepsilon$. Numerical methods that act uniformly well for all the values of the singular perturbation parameter are called $\varepsilon$-uniformly convergent numerical methods.

Many authors have worked on the numerical solution of the problem 1.1a)(1.1b with different assumptions about the function $f$, as well as more general nonlinear problems. There were many constructed $\varepsilon$-uniformly convergent difference schemes of order 2 and higher (Herceg [2, Herceg, Surla and Rapajić [3], Herceg and Miloradović [4], Herceg and Herceg [5], Kopteva and Linß [8, Kopteva and Stynes [9, 10], Kopteva, Pickett and Purtill [11], Linß, Roos and Vulanović [13], Sun and Stynes [21, Stynes and Kopteva [22, Surla and Uzelac [23, Vulanović [24, 25, 26, 27, 29], etc.

In the paper [1] Boglaev introduced a new method for finding the numerical solution of the problem $(1.1 \mathrm{a})-(1.1 \mathrm{~b})$, using the representation of the exact problem to $1.1 \mathrm{a}-1.1 \mathrm{~b}$ via the Green function. In this paper we use this method to construct a new different scheme.

\section{THEORETICAL BACKGROUND}

The estimates of solution's derivatives are a very important tool in the analysis of numerical methods considering the singularly-perturbed boundary-value problems. The construction of layer-adapted meshes is based on these estimates, also in the sequel they will be used in the analysis of the consistency. Bearing in mind the above, we state the following theorem about a decomposition of the solution $y$ to a layer component $s$ and a regular component $r$ and the appropriate estimates.

Theorem 1. [24] The solution y to problem $1.1 \mathrm{a}-1.1 \mathrm{~b}$ can be represented in the following way:

$$
y=r+s,
$$

where for $j=0,1, \ldots, k+2$ and $x \in[0,1]$ the following inequalities hold

$$
\left|r^{(j)}(x)\right| \leq C
$$

and

$$
\left|s^{(j)}(x)\right| \leq C \varepsilon^{-j}\left(e^{-\frac{x}{\varepsilon} \sqrt{m}}+e^{-\frac{1-x}{\varepsilon} \sqrt{m}}\right) .
$$

2.1. Layer-adapted mesh. It's a well-known that the exact solution to problems like $1.1 \mathrm{a}-1.1 \mathrm{~b}$ changes rapidly near the end points $x=0$ and $x=1$. Many meshes have been constructed for finding the numerical solution of problems that have a layer or layers of an exponential type. In the present paper we shall use three different meshes. We will get these meshes $0=x_{0}<x_{1}<\ldots<x_{N}=1$, by using appropriate generating functions, i.e. $x_{i}=\psi(i / N)$. The generating function are constructed as follows. 
Let $N+1$ be the number of mesh points, $q \in(0,1 / 2)$ mesh parameter. Define the Shishkin mesh transition point by

$$
\lambda:=\min \left\{\frac{2 \varepsilon \ln N}{\sqrt{m}}, \frac{1}{4}\right\} .
$$

The first mesh we will use in the sequel is a modified Shishkin mesh proposed by Vulanović [28]. The generating function for this mesh is

$$
\psi(t)=\left\{\begin{array}{l}
4 \lambda t, \quad t \in[0,1 / 4], \\
p(t-1 / 4)^{3}+4 \lambda t, \quad t \in[1 / 4,1 / 2], \\
1-\psi(1-t), \quad t \in[1 / 2,1],
\end{array}\right.
$$

where $p$ is chosen so that $\psi(1 / 2)=1 / 2$, i.e. $p=32(1-4 \lambda)$. Note that $\psi \in C^{1}[0,1]$ with $\left\|\psi^{\prime}\right\|_{\infty} \leqslant C,\left\|\psi^{\prime \prime}\right\|_{\infty} \leqslant C$. Therefore the mesh size $h_{i}=x_{i+1}-x_{i}, i=0, \ldots N-1$ satisfies (see [14])

$$
\begin{aligned}
h_{i} & =\int_{i / N}^{(i+1) N} \psi^{\prime}(t) \mathrm{d} t \leqslant C N^{-1}, \\
\left|h_{i+1}-h_{i}\right| & =\left|\int_{(i-1) / N}^{i / N} \int_{t}^{t+1 / N} \psi^{\prime \prime}(s) \mathrm{d} s\right| \leqslant C N^{-2} .
\end{aligned}
$$

The second mesh is the Shishkin mesh [20]. The generating function for this mesh is

$$
\psi(t)=\left\{\begin{array}{l}
4 \lambda t, \quad t \in[0,1 / 4] \\
\lambda+2(1-2 \lambda)(t-1 / 4), \quad t \in[1 / 2,1 / 4] \\
1-\psi(1-t), \quad t \in[1 / 2,1] .
\end{array}\right.
$$

The third mesh is the modified Bakhvalov mesh also proposed by Vulanović [24]. The generating function for this mesh is

$$
\psi(t)=\left\{\begin{array}{l}
\mu(t):=\frac{a \varepsilon t}{q-t}, \quad t \in[0, \alpha] \\
\mu(\alpha)+\mu^{\prime}(\alpha)(t-\alpha), \quad t \in[\alpha, 1 / 2] \\
1-\psi(1-t), \quad t \in[1 / 2,1]
\end{array}\right.
$$

where $a$ and $q$ are constants, independent of $\varepsilon$, such that $q \in(0,1 / 2), a \in(0, q / \varepsilon)$, and additionally $a \sqrt{m} \geqslant 2$. The parameter $\alpha$ is the abscissa of the contact point of the tangent line from $(1 / 2,1 / 2)$ to $\mu(t)$, and its value is

$$
\alpha=\frac{q-\sqrt{a q \varepsilon(1-2 q+2 a \varepsilon)}}{1+2 a \varepsilon} .
$$

The fourth mesh is proposed by Liseikin [15, 16], and we will use its modification from [17. The generating function for this mesh is 


$$
\psi(t)= \begin{cases}c_{1} \varepsilon^{k}\left((1-d t)^{-1 / a}-1\right), & 0 \leqslant t \leqslant 1 / 4 \\ c_{1}\left[\varepsilon^{k a n /(1+n a)}-\varepsilon^{k}+d \frac{1}{a} \varepsilon^{k a(n-1) /(1+n a)}(t-1 / 4)\right. & \\ +\frac{1}{2} d^{2} \frac{1}{a}\left(\frac{1}{a}+1\right) \varepsilon^{k a(n-2) /(1+n a)}(t-1 / 4)^{2} & \\ \left.+c_{0}(t-1 / 4)^{3}\right], & 1 / 4 \leqslant t \leqslant 1 / 2, \\ 1-\psi(1-t), & 1 / 2 \leqslant t \leqslant 1\end{cases}
$$

where $d=\left(1-\varepsilon^{k a /(1+n a)}\right) /(1 / 4), a$ is a positive constant subject to $a \geq m_{1}>0$, and $a=1, c_{0}>0, n=2, k=1, c_{0}=0$, and $\frac{1}{c_{1}}=2\left[\varepsilon^{k a n /(1+n a)}-\varepsilon^{k}\right.$ $\left.+\frac{d}{4 a} \varepsilon^{k a(n-1) /(1+n a)}+\frac{d^{2}}{2} \frac{1}{a}\left(\frac{1}{a}+1\right) \varepsilon^{k a(n-2) /(1+n a)}(1 / 4)^{2}+c_{0}(1 / 4)^{3}\right]$ is chosen here.

\section{Difference SCheme}

We will consider an arbitrary mesh with mesh points

$$
0=x_{0}<x_{1}<\ldots<x_{N}=1,
$$

and let $h_{i}=x_{i+1}-x_{i}, i=0,1, \ldots, N-1$. In constructing a new difference scheme for the problem (1.1a)-1.1b) the following scheme from Boglaev [1] will be used

$$
\begin{array}{r}
\frac{\beta}{\sinh \left(\beta h_{i-1}\right)} y_{i-1}-\left(\frac{\beta}{\tanh \left(\beta h_{i-1}\right)}+\frac{\beta}{\tanh \left(\beta h_{i}\right)}\right) y_{i}+\frac{\beta}{\sinh \left(\beta h_{i}\right)} y_{i+1} \\
=\frac{1}{\varepsilon^{2}}\left[\int_{x_{i-1}}^{x_{i}} u_{i-1}^{I I} \psi(s, y) \mathrm{d} s+\int_{x_{i}}^{x_{i+1}} u_{i}^{I} \psi(s, y) \mathrm{d} s\right]
\end{array}
$$

$i=1,2, \ldots, N-1, y_{0}=y_{N}=0$, where

$$
\psi(s, y)=f(s, y)-\gamma y, \beta=\frac{\sqrt{\gamma}}{\varepsilon},
$$

and the functions $u_{i}^{I}, u_{i}^{I I}$ are the solutions of the boundary-value problems

$$
\begin{aligned}
& \varepsilon^{2} u_{i}^{\prime \prime}-\gamma u_{i}=0 \text { on }\left(x_{i}, x_{i+1}\right), \\
& u_{i}\left(x_{i}\right)=1, u_{i}\left(x_{i+1}\right)=0, \\
& i=0,1, \ldots, N-1, \\
& \begin{array}{c}
\varepsilon^{2} u_{i}^{\prime \prime}-\gamma u_{i}=0 \text { on }\left(x_{i}, x_{i+1}\right) \\
u_{i}\left(x_{i}\right)=0, u_{i}\left(x_{i+1}\right)=1, \\
i=0,1, \ldots, N-1,
\end{array}
\end{aligned}
$$

respectively.

We can't calculate the integrals in (3.1) because we don't know the exact solution $y$ to the problem 1.1a $1.1 \mathrm{~b}$. The next step is to approximate the function $\psi$ by a constant value. Approximations of the function $\psi$ are

$$
\begin{aligned}
& \psi_{i}^{-}=(1-t) \psi\left(x_{i-1}, y\left(x_{i-1}\right)\right)+t \psi\left(x_{i}, y\left(x_{i}\right)\right), x \in\left[x_{i-1}, x_{i}\right], \\
& \psi_{i}^{+}=t \psi\left(x_{i}, y\left(x_{i}\right)\right)+(1-t) \psi\left(x_{i+1}, y\left(x_{i+1}\right)\right), x \in\left[x_{i}, x_{i+1}\right], t \in[0,1] .
\end{aligned}
$$


By using the approximations (3.3), (3.4) into (3.1), after calculating the integrals and some computing, and taking in account that

$$
\begin{gathered}
\int_{x_{i-1}}^{x_{i}} u_{i-1}^{I I} \mathrm{~d} s=\frac{\cosh \left(\beta h_{i-1}\right)-1}{\beta \sinh \left(\beta h_{i-1}\right)}, \\
\int_{x_{i}}^{x_{i+1}} u_{i}^{I} \mathrm{~d} s=\frac{\cosh \left(\beta h_{i}\right)-1}{\beta \sinh \left(\beta h_{i}\right)},
\end{gathered}
$$

we get the difference scheme

$$
\begin{aligned}
& \frac{(1-t) \cosh \left(\beta h_{i-1}\right)+t}{\sinh \left(\beta h_{i-1}\right)}\left(\bar{y}_{i-1}-\bar{y}_{i}\right)-\frac{(1-t) \cosh \left(\beta h_{i}\right)+t}{\sinh \left(\beta h_{i}\right)}\left(\bar{y}_{i}-\bar{y}_{i+1}\right) \\
& -\frac{(1-t) f_{i-1}+t f_{i}}{\gamma} \cdot \frac{\cosh \left(\beta h_{i-1}\right)-1}{\sinh \left(\beta h_{i-1}\right)}-\frac{t f_{i}+(1-t) f_{i+1}}{\gamma} \cdot \frac{\cosh \left(\beta h_{i}\right)-1}{\sinh \left(\beta h_{i}\right)}=0
\end{aligned}
$$

where $i=1,2, \ldots, N-1, f_{k}=f\left(x_{k}, \bar{y}_{k}\right), k \in\{i-1, i, i+1\}$, and $t \in[0,1]$.

\section{Stability}

The difference scheme (3.5) generates a nonlinear system. A goal of this section is to show that this system has a unique solution. We are going to construct a discrete operator $T$, and show that this operator is inverse-monotone as well, which implies that our numerical method is stable, and the numerical solution exists and it is unique.

Let us set the discrete operator

$$
T u=\left(T u_{0}, T u_{1}, \ldots, T u_{N}\right)^{T},
$$

where

$$
\begin{aligned}
T u_{0}= & -u_{0}, \quad \gamma \\
T u_{i}= & \frac{\cosh \left(\beta h_{i-1}\right)-1}{\sinh \left(\beta h_{i-1}\right)}+\frac{\cosh \left(\beta h_{i}\right)-1}{\sinh \left(\beta h_{i}\right)} \\
& \cdot\left\{\frac{(1-t) \cosh \left(\beta h_{i-1}\right)+t}{\sinh \left(\beta h_{i-1}\right)}\left(\bar{u}_{i-1}-\bar{u}_{i}\right)-\frac{(1-t) \cosh \left(\beta h_{i}\right)+t}{\sinh \left(\beta h_{i}\right)}\left(\bar{u}_{i}-\bar{u}_{i+1}\right)\right. \\
& \left.-\frac{(1-t) f_{i-1}+t f_{i}}{\gamma} \cdot \frac{\cosh \left(\beta h_{i-1}\right)-1}{\sinh \left(\beta h_{i-1}\right)}-\frac{t f_{i}+(1-t) f_{i+1}}{\gamma} \cdot \frac{\cosh \left(\beta h_{i}\right)-1}{\sinh \left(\beta h_{i}\right)}\right\} \\
& =0, \quad i=1, \ldots, N-1, \\
T u_{N}= & -u_{N}, \text { and } f_{k}=f\left(x_{k}, u_{k}\right), k \in\{i-1, i, i+1\} .
\end{aligned}
$$

Obviously, it is true that

$$
T \bar{y}=0
$$

where $\bar{y}=\left(\bar{y}_{0}, \bar{y}_{1}, \ldots, \bar{y}_{N}\right)^{T}$ is the numerical solution of the problem 1.1a $1.1 \mathrm{~b}$, obtained by using the difference scheme (3.5). Now, we can state and prove the theorem of stability. 
Theorem 2. The discrete problem (4.1) -4.3) has a unique solution $\bar{y}$ for $\gamma \geqslant f_{y}$. Moreover, for every $v, w \in \mathbb{R}^{N+1}$ we have the following stability inequality

$$
\|v-w\| \leqslant C\|T v-T w\| .
$$

Proof. We use a well known technique from [27] to prove the first statement of the theorem. The proof of existence and uniqueness of the solution of the discrete problem $T y=0$ is based on the proof of the relation: $\left\|\left(T^{\prime} y\right)^{-1}\right\| \leqslant C$, where $T^{\prime}$ is the Fréchet derivative of $T$. The Fréchet derivative $H:=T^{\prime}(y)$ is a tridiagonal matrix. Let $H=\left[h_{i j}\right]$. The non-zero elements of this tridiagonal matrix are

$$
\begin{aligned}
h_{1,1}=h_{N+1, N+1}=-1<0, & {\left[\frac{(1-t) \cosh \left(\beta h_{i-1}\right)+t}{\sinh \left(\beta h_{i-1}\right)}-\frac{\frac{\partial f}{\partial y_{i-1}}}{\gamma} \cdot \frac{(1-t)\left(\cosh \left(\beta h_{i-1}\right)-1\right)}{\sinh \left(\beta h_{i-1}\right)}\right], } \\
h_{i, i-1}=\Lambda \cdot & {\left[\frac{(1-t) \cosh \left(\beta h_{i}\right)+t}{\sinh \left(\beta h_{i}\right)}-\frac{\partial f}{\partial y_{i+1}} \cdot \frac{(1-t)\left(\cosh \left(\beta h_{i}\right)-1\right)}{\sinh \left(\beta h_{i}\right)}\right], } \\
h_{i, i+1}=\Lambda \cdot & {\left[\frac{(1-t) \cosh \left(\beta h_{i-1}\right)+t}{\sinh \left(\beta h_{i-1}\right)}+\frac{(1-t) \cosh \left(\beta h_{i}\right)+t}{\sinh \left(\beta h_{i}\right)}\right.} \\
h_{i, i}=-\Lambda \cdot & \left.\frac{\partial f}{\partial y_{i}} \cdot \frac{\cosh \left(\beta h_{i-1}\right)-1}{\sinh \left(\beta h_{i-1}\right)}+t \frac{\frac{\partial f}{\partial y_{i}}}{\gamma} \cdot \frac{\cosh \left(\beta h_{i}\right)-1}{\sinh \left(\beta h_{i}\right)}\right], i=2, \ldots, N,
\end{aligned}
$$

where $\Lambda=\frac{\gamma}{\frac{\cosh \left(\beta h_{i-1}\right)-1}{\sinh \left(\beta h_{i-1}\right)}+\frac{\cosh \left(\beta h_{i}\right)-1}{\sinh \left(\beta h_{i}\right)}}$. From 4.5, it's obvious that

$$
h_{i, i-1}>0, h_{i, i+1}>0, h_{i, i}<0,
$$

and

$$
\left|h_{i, i}\right|-\left|h_{i, i-1}\right|-\left|h_{i, i+1}\right| \geqslant m,
$$

so we can conclude that $H$ is an $M$-matrix, and finally we obtain

$$
\left\|H^{-1}\right\| \leqslant \frac{1}{m}
$$

Using Hadamard's theorem ([19, p 137]) we get that $T$ is a homeomorphism. Since clearly $\mathbb{R}^{N+1}$ is non-empty and 0 is the only image of the mapping $T$, we have that 4.3 has a unique solution.

The proof of second statement of the Theorem 4.4 is based on a part of the proof of Theorem 3 from [2]. The following is true $T v-T w=\left(T^{\prime} \xi\right)^{-1}(v-w)$ for some $\xi=\left(\xi_{0}, \xi_{1}, \ldots, \xi_{N}\right)^{T} \in \mathbb{R}^{N+1}$. Therefore $v-w=\left(T^{\prime} \xi\right)^{-1}(T v-T w)$ and finally due to inequality 4.6 we have that

$$
\|v-w\|=\left\|\left(T^{\prime} \xi\right)^{-1}(T v-T w)\right\| \leqslant \frac{1}{m}\|T v-T w\| .
$$




\section{UNIFORM CONVERGENCE}

The difference scheme 3.5 can be written in the following form

$$
\begin{gathered}
(1-t)\left[\frac{\cosh \left(\beta h_{i-1}\right)-1}{\sinh \left(\beta h_{i-1}\right)}\left(\bar{y}_{i-1}-\bar{y}_{i}\right)-\frac{\cosh \left(\beta h_{i}\right)-1}{\sinh \left(\beta h_{i}\right)}\left(\bar{y}_{i}-\bar{y}_{i+1}\right)\right] \\
+\frac{\bar{y}_{i-1}-\bar{y}_{i}}{\sinh \left(\beta h_{i-1}\right)}-\frac{\bar{y}_{i}-\bar{y}_{i+1}}{\sinh \left(\beta h_{i}\right)} \\
\left.-\frac{(1-t) f_{i-1}+t f_{i}}{\gamma} \cdot \frac{\cosh \left(\beta h_{i-1}\right)-1}{\sinh \left(\beta h_{i-1}\right)}-\frac{t f_{i}+(1-t) f_{i+1}}{\gamma} \cdot \frac{\cosh \left(\beta h_{i}\right)-1}{\sinh \left(\beta h_{i}\right)}\right]=0,
\end{gathered}
$$

$i=1, \ldots, N-1$.

In order to prove the Theorem of convergence, we need three estimates given in the next lemmas.

Lemma 1. [6] Assume that $\varepsilon \leqslant \frac{C}{N}$. In the part of the modified Shishkin mesh from Section 2.1 when $x_{i}, x_{i \pm 1} \in\left[x_{N / 4-1}, \lambda\right] \cup[\lambda, 1 / 2]$, we have the following estimate $(i=N / 4, \ldots, N / 2-1)$

$$
\left|\frac{\frac{\cosh \left(\beta h_{i-1}\right)-1}{\sinh \left(\beta h_{i-1}\right)}\left(y\left(x_{i-1}\right)-y\left(x_{i}\right)\right)-\frac{\cosh \left(\beta h_{i}\right)-1}{\sinh \left(\beta h_{i}\right)}\left(y\left(x_{i}\right)-y\left(x_{i+1}\right)\right)}{\frac{\cosh \left(\beta h_{i-1}\right)-1}{\sinh \left(\beta h_{i-1}\right)}+\frac{\cosh \left(\beta h_{i}\right)-1}{\sinh \left(\beta h_{i}\right)}}\right| \leqslant \frac{C}{N^{2}} .
$$

Lemma 2. [6] Assume that $\varepsilon \leqslant \frac{C}{N}$. In the part of the modified Shishkin mesh from Section 2.1 when $x_{i}, x_{i \pm 1} \in\left[x_{N / 4-1}, \lambda\right] \cup[\lambda, 1 / 2]$, we have the following estimate

$$
\left|\frac{\frac{y\left(x_{i-1}\right)-y\left(x_{i}\right)}{\sinh \left(\beta h_{i-1}\right)}-\frac{y\left(x_{i}\right)-y\left(x_{i+1}\right)}{\sinh \left(\beta h_{i}\right)}}{\frac{\cosh \left(\beta h_{i-1}\right)-1}{\sinh \left(\beta h_{i-1}\right)}+\frac{\cosh \left(\beta h_{i}\right)-1}{\sinh \left(\beta h_{i}\right)}}\right| \leqslant \frac{C}{N^{2}}, i=N / 4, \ldots, N / 2-1 .
$$

Lemma 3. Assume that $\varepsilon \leqslant \frac{C}{N}$. In the part of the modified Shishkin mesh from Section 2.1 when $x_{i}, x_{i \pm 1} \in\left[x_{N / 4-1}, \lambda\right] \cup[\lambda, 1 / 2]$, we have the following estimate

$$
\begin{aligned}
& \frac{\gamma}{\frac{\cosh \left(\beta h_{i-1}\right)-1}{\sinh \left(\beta h_{i-1}\right)}+\frac{\cosh \left(\beta h_{i}\right)-1}{\sinh \left(\beta h_{i}\right)}} \mid \frac{(1-t) f\left(x_{i-1}, y\left(x_{i-1}\right)\right)+t f\left(x_{i}, y\left(x_{i}\right)\right)}{\gamma} \\
& \cdot \frac{\cosh \left(\beta h_{i-1}\right)-1}{\sinh \left(\beta h_{i-1}\right)}-\frac{t f\left(x_{i}, y\left(x_{i}\right)\right)+(1-t) f\left(x_{i+1}, y\left(x_{i+1}\right)\right)}{\gamma} \cdot \frac{\cosh \left(\beta h_{i}\right)-1}{\sinh \left(\beta h_{i}\right)} \mid \\
& \leqslant \frac{C}{N^{2}}, i=N / 4, \ldots, N / 2-1 .
\end{aligned}
$$

Proof. Taking into consideration the assumption $\varepsilon \leqslant \frac{C}{N}$, the equality $\varepsilon^{2} y^{\prime \prime}\left(x_{i}\right)=$ $f\left(x_{i}, y\left(x_{i}\right)\right)$, and the Theorem of decomposition, we obtain

$$
\begin{aligned}
& \frac{\gamma}{\frac{\cosh \left(\beta h_{i-1}\right)-1}{\sinh \left(\beta h_{i-1}\right)}+\frac{\cosh \left(\beta h_{i}\right)-1}{\sinh \left(\beta h_{i}\right)}} \mid \frac{(1-t) f\left(x_{i-1}, y\left(x_{i-1}\right)\right)+t f\left(x_{i}, y\left(x_{i}\right)\right)}{\gamma} \\
& \cdot \frac{\cosh \left(\beta h_{i-1}\right)-1}{\sinh \left(\beta h_{i-1}\right)}-\frac{t f\left(x_{i}, y\left(x_{i}\right)\right)+(1-t) f\left(x_{i+1}, y\left(x_{i+1}\right)\right)}{\gamma} \cdot \frac{\cosh \left(\beta h_{i}\right)-1}{\sinh \left(\beta h_{i}\right)} \mid
\end{aligned}
$$




$$
\begin{aligned}
& \leqslant\left|(1-t) f\left(x_{i-1}, y\left(x_{i-1}\right)\right)+2 t f\left(x_{i}, y\left(x_{i}\right)\right)+(1-t) f\left(x_{i+1}, y\left(x_{i+1}\right)\right)\right| \\
& \leqslant \varepsilon^{2}\left[(1-t)\left(\left|r^{\prime \prime}\left(x_{i-1}\right)\right|+\left|s^{\prime \prime}\left(x_{i-1}\right)\right|\right)\right. \\
& \left.+2 t\left(\left|s^{\prime \prime}\left(x_{i}\right)\right|+\left|r^{\prime \prime}\left(x_{i}\right)\right|\right)+(1-t)\left(\left|s^{\prime \prime}\left(x_{i+1}\right)\right|+\left|r^{\prime \prime}\left(x_{i+1}\right)\right|\right)\right] \\
& \leqslant C_{1} \varepsilon^{2}\left[(1-t)\left(2+\frac{e^{-\frac{x_{i-1}}{\varepsilon} \sqrt{m}}}{\varepsilon^{2}}+\frac{e^{-\frac{x_{i+1}}{\varepsilon} \sqrt{m}}}{\varepsilon^{2}}\right)+2 t\left(1+\frac{e^{-\frac{x_{i}}{\varepsilon} \sqrt{m}}}{\varepsilon^{2}}\right)\right] \\
& \leqslant C\left(\varepsilon^{2}+\frac{1}{N^{2}}\right), i=N / 4, \ldots, N / 2-1 .
\end{aligned}
$$

Theorem 3. The discrete problem (4.1)-4.3) on the modified Shishkin mesh 2.4) from Section 2.1 is uniformly convergent with respect to $\varepsilon$ and

$$
\max _{i}\left|y\left(x_{i}\right)-\bar{y}_{i}\right| \leqslant C \begin{cases}\left(\ln ^{2} N\right) / N^{2}, & i=0, \ldots, N / 4-1, \\ 1 / N^{2}, & i=N / 4, \ldots, 3 N / 4, \\ \left(\ln ^{2} N\right) / N^{2}, & i=3 N / 4+1, \ldots, N,\end{cases}
$$

where $y\left(x_{i}\right)$ is the value of the exact solution, $\bar{y}_{i}$ is the value of the numerical solution of the problem 1.1a) 1.1b in the mesh point $x_{i}$, respectively, and $C>0$ is a constant independent of $N$ and $\varepsilon$.

Proof.

Case $0 \leqslant i<N / 4-1$. Here $h_{i-1}=h_{i}$ and $h_{i}=\mathcal{O}(\varepsilon \ln N / N)$, thus we have

$$
\begin{aligned}
& (T y)_{i}=\frac{\gamma}{\frac{\cosh \left(\beta h_{i-1}\right)-1}{\sinh \left(\beta h_{i-1}\right)}+\frac{\cosh \left(\beta h_{i}\right)-1}{\sinh \left(\beta h_{i}\right)}} \\
& {\left[\frac{(1-t) \cosh \left(\beta h_{i-1}\right)+t}{\sinh \left(\beta h_{i-1}\right)}\left(y\left(x_{i-1}\right)-y\left(x_{i}\right)\right)\right.} \\
& -\frac{(1-t) \cosh \left(\beta h_{i}\right)+t}{\sinh \left(\beta h_{i}\right)}\left(y\left(x_{i}\right)-y\left(x_{i+1}\right)\right) \\
& -\frac{(1-t) f\left(x_{i-1}, y\left(x_{i-1}\right)\right)+t f\left(x_{i}, y\left(x_{i}\right)\right)}{\gamma} \cdot \frac{\cosh \left(\beta h_{i-1}\right)-1}{\sinh \left(\beta h_{i-1}\right)} \\
& \left.\quad-\frac{t f\left(x_{i}, y\left(x_{i}\right)\right)+(1-t) f\left(x_{i+1}, y\left(x_{i+1}\right)\right)}{\gamma} \cdot \frac{\cosh \left(\beta h_{i}\right)-1}{\sinh \left(\beta h_{i}\right)}\right] \\
& =\frac{\gamma \quad\left\{t \left[y\left(x_{i-1}\right)-2 y\left(x_{i}\right)+y\left(x_{i+1}\right)\right.\right.}{2\left(\cosh \left(\beta h_{i}\right)-1\right)} \\
& \left.\quad-\frac{2 f\left(x_{i}, y\left(x_{i}\right)\right)}{\gamma}\left(\cosh \left(\beta h_{i}\right)-1\right)\right] \\
& +(1-t)\left[\cosh \left(\beta h_{i}\right)\left(y\left(x_{i-1}\right)-2 y\left(x_{i}\right)+y\left(x_{i+1}\right)\right)\right.
\end{aligned}
$$


ON CONSTRUCTION OF A GLOBAL NUMERICAL SOLUTION...

$$
\begin{gathered}
\left.\left.-\frac{f\left(x_{i-1}, y\left(x_{i-1}\right)\right)+f\left(x_{i+1}, y\left(x_{i+1}\right)\right)}{\gamma} \cdot\left(\cosh \left(\beta h_{i}\right)-1\right)\right]\right\} \\
=\frac{\gamma}{2\left(\cosh \left(\beta h_{i}\right)-1\right)} \\
\left\{t\left[y\left(x_{i-1}\right)-2 y\left(x_{i}\right)+y\left(x_{i+1}\right)-\frac{2 \varepsilon^{2} y^{\prime \prime}\left(x_{i}\right)}{\gamma}\left(\cosh \left(\beta h_{i}\right)-1\right)\right]\right. \\
+(1-t)\left[\cosh \left(\beta h_{i}\right)\left(y\left(x_{i-1}\right)-2 y\left(x_{i}\right)+y\left(x_{i+1}\right)\right)\right. \\
\left.\left.-\varepsilon^{2} \frac{y^{\prime \prime}\left(x_{i-1}\right)+y^{\prime \prime}\left(x_{i+1}\right)}{\gamma} \cdot\left(\cosh \left(\beta h_{i}\right)-1\right)\right]\right\} .
\end{gathered}
$$

Using Taylor's expansions

$$
\begin{aligned}
& y\left(x_{i-1}\right)-2 y\left(x_{i}\right)+y\left(x_{i+1}\right)=y^{\prime \prime}\left(x_{i}\right) h_{i}^{2}+\frac{y^{(i v)}\left(\xi_{i}^{-}\right)+y^{(i v)}\left(\xi_{i}^{+}\right)}{24} h_{i}^{4}, \\
& y^{\prime \prime}\left(x_{i-1}\right)+y^{\prime \prime}\left(x_{i+1}\right)=2 y^{\prime \prime}\left(x_{i}\right)+\frac{y^{(i v)}\left(\eta_{i}^{-}\right)+y^{(i v)}\left(\eta_{i}^{+}\right)}{2} h_{i}^{2}, \\
& \cosh \left(\beta h_{i}\right)=1+\frac{\beta^{2} h_{i}^{2}}{2}+\mathcal{O}\left(\beta^{4} h_{i}^{4}\right),
\end{aligned}
$$

$\xi_{i}^{-} \in\left(x_{i-1}, x_{i}\right), \xi_{i}^{+} \in\left(x_{i}, x_{i+1}\right), \eta_{i}^{-} \in\left(x_{i-1}, x_{i}\right), \eta_{i}^{+} \in\left(x_{i}, x_{i+1}\right)$, we get

$$
\begin{aligned}
& (T y)_{i}=\frac{\gamma \cdot t}{\beta^{2} h_{i}^{2}+2 \mathcal{O}\left(\beta^{4} h_{i}^{4}\right)}\left[y^{\prime \prime}\left(x_{i}\right) h_{i}^{2}+\frac{y^{(i v)}\left(\xi_{i}^{-}\right)+y^{(i v)}\left(\xi_{i}^{+}\right)}{24} h_{i}^{4}\right. \\
& \left.-\frac{2 \varepsilon^{2} y^{\prime \prime}\left(x_{i}\right)}{\gamma}\left(\frac{\beta^{2} h_{i}^{2}}{2}+\mathcal{O}\left(\beta^{4} h_{i}^{4}\right)\right)\right] \\
& +\frac{\gamma \cdot(1-t)}{\beta^{2} h_{i}^{2}+2 \mathcal{O}\left(\beta^{4} h_{i}^{4}\right)}\left[\left(y^{\prime \prime}\left(x_{i}\right) h_{i}^{2}+\frac{y^{(i v)}\left(\xi_{i}^{-}\right)+y^{(i v)}\left(\xi_{i}^{+}\right)}{24} h_{i}^{4}\right)\right. \\
& \cdot\left(1+\frac{\beta^{2} h_{i}^{2}}{2}+\mathcal{O}\left(\beta^{4} h_{i}^{4}\right)\right) \\
& \left.-\varepsilon^{2} \frac{2 y^{\prime \prime}\left(x_{i}\right)+\frac{y^{(i v)}\left(\eta_{i}^{-}\right)+y^{(i v)}\left(\eta_{i}^{+}\right)}{2}}{\gamma}\left(\frac{\beta^{2} h_{i}^{2}}{2}+\mathcal{O}\left(\beta^{4} h_{i}^{4}\right)\right)\right] \\
& =\frac{\gamma \cdot t}{\beta^{2} h_{i}^{2}+2 \mathcal{O}\left(\beta^{4} h_{i}^{4}\right)}\left[\frac{y^{(i v)}\left(\xi_{i}^{-}\right)+y^{(i v)}\left(\xi_{i}^{+}\right)}{24} h_{i}^{4}-\frac{2 \varepsilon^{2} y^{\prime \prime}\left(x_{i}\right)}{\gamma} \mathcal{O}\left(\beta^{4} h_{i}^{4}\right)\right] \\
& +\frac{\gamma \cdot(1-t)}{\beta^{2} h_{i}^{2}+2 \mathcal{O}\left(\beta^{4} h_{i}^{4}\right)}\left[\frac{y^{(i v)}\left(\xi_{i}^{-}\right)+y^{(i v)}\left(\xi_{i}^{+}\right)}{24} h_{i}^{4}\right. \\
& +\left(\frac{\beta^{2} h_{i}^{2}}{2}+\mathcal{O}\left(\beta^{4} h_{i}^{4}\right)\right)\left(y^{\prime \prime}\left(x_{i}\right) h_{i}^{2}+\frac{y^{(i v)}\left(\xi_{i}^{-}\right)+y^{(i v)}\left(\xi_{i}^{+}\right)}{24} h_{i}^{4}\right) \\
& -\frac{2 \varepsilon^{2} y^{\prime \prime}\left(x_{i}\right)}{\gamma} \cdot \mathcal{O}\left(\beta^{4} h_{i}^{4}\right) \\
& \left.+\varepsilon^{2} \frac{y^{(i v)}\left(\eta_{i}^{-}\right)+y^{(i v)}\left(\eta_{i}^{+}\right)}{2 \gamma} h_{i}^{2}\left(\frac{\beta^{2} h_{i}^{2}}{2}+\mathcal{O}\left(\beta^{4} h_{i}^{4}\right)\right)\right],
\end{aligned}
$$


and finally

$$
\left|(T y)_{i}\right| \leqslant\left(C \ln ^{2} N\right) / N^{2}, i=0,1, \ldots, N / 4-1 .
$$

Case $N / 4 \leqslant i<N / 2$. Due to (5.1) we have the next inequality

$$
\begin{aligned}
\left|(T y)_{i}\right| \leqslant & \frac{\gamma}{\frac{\cosh \left(\beta h_{i-1}\right)-1}{\sinh \left(\beta h_{i-1}\right)}+\frac{\cosh \left(\beta h_{i}\right)-1}{\sinh \left(\beta h_{i}\right)}} \\
& \cdot\left[(1-t) \mid \frac{\cosh \left(\beta h_{i-1}\right)-1}{\sinh \left(\beta h_{i-1}\right)}\left(y\left(x_{i-1}\right)-y\left(x_{i}\right)\right)\right. \\
& +\left|\frac{y\left(x_{i-1}\right)-y\left(x_{i}\right)}{\sinh \left(\beta h_{i-1}\right)}-\frac{y\left(x_{i}\right)-y\left(x_{i+1}\right)}{\sinh \left(\beta h_{i}\right)}\right| \\
& +\left|\frac{(1-t) f\left(x_{i-1}, y\left(x_{i-1}\right)\right)+t f\left(x_{i}, y\left(x_{i}\right)\right)}{\gamma} \cdot \frac{\cosh \left(\beta h_{i-1}\right)-1}{\sinh \left(\beta h_{i-1}\right)}\right| \\
& \left.+\left|\frac{t f\left(x_{i}, y\left(x_{i}\right)\right)+(1-t) f\left(x_{i+1}, y\left(x_{i+1}\right)\right)}{\gamma} \cdot \frac{\cosh \left(\beta h_{i}\right)-1}{\sinh \left(\beta h_{i}\right)}\right|\right],
\end{aligned}
$$

and according to Lemma 1 Lemma 2 and Lemma 3 we obtain

$$
\left|(T y)_{i}\right| \leqslant C / N^{2}, i=N / 4, \ldots, N / 2-1 .
$$

Case $i=N / 2$. This case is trivial, because $h_{N / 4-1}=h_{N / 4}$ and the influence of the layer component $s$ is negligible.

Collecting (5.5), (5.6) and taking into account Case $i=N / 2$, we have proven the theorem.

\section{Global solution}

In the paper [7 a global numerical solution was constructed, using a spline in tension, and the authors proved the uniform convergence of order 1 for this solution on the modified Shishkin mesh generated by (2.4). After that, they repaired the global numerical solution on $[\lambda, 1-\lambda]$ and achieved the uniform convergence of order 2. That repaired global solution is composed of exponential and linear functions. In the sequel we avoid exponential functions and give a global numerical solution composed by linear functions. We will also include a global solution obtained by using a natural cubic spline in the numerical experiments, because this spline is the lowest degree spline with a continuous second derivative. 
Linear spline. Let the global numerical solution to the problem $1.1 \mathrm{a}-1.1 \mathrm{~b}$ has the form

$$
\bar{P}(x)=\left\{\begin{array}{cl}
\bar{p}_{1}(x), & x \in\left[x_{0}, x_{1}\right], \\
\bar{p}_{2}(x), & x \in\left[x_{1}, x_{2}\right], \\
\vdots & \\
\bar{p}_{i}(x), & x \in\left[x_{i-1}, x_{i}\right], \\
\vdots & \\
\bar{p}_{N}(x), & x \in\left[x_{N-1}, x_{N}\right],
\end{array}\right.
$$

where

$$
\bar{p}_{i}(x)=\left\{\begin{array}{cc}
\frac{\bar{y}_{i}-\bar{y}_{i-1}}{x_{i}-x_{i-1}}\left(x-x_{i-1}\right)+\bar{y}_{i-1}, & x \in\left[x_{i-1}, x_{i}\right], \\
0, & x \notin\left[x_{i-1}, x_{i}\right],
\end{array}\right.
$$

and $i=1,2, \ldots, N$.

Theorem 4. The following estimate of the error holds

$$
\max _{x \in[0,1]}|y(x)-\bar{P}(x)| \leqslant\left(C \ln ^{2} N\right) / N^{2},
$$

where $y$ is the exact solution to the problem $1.1 \mathrm{a}-1.1 \mathrm{~b}$ and $\bar{P}$ is the global numerical solution (6.1).

Proof. We divide this proof in three parts, $[0, \lambda],\left[\lambda, x_{N / 4+1}\right]$ and $\left[x_{N / 4+1}, 1 / 2\right]$. The proof is analogues on $[1 / 2,1]$. The proof is based on the inequality $\| \bar{P}-$ $y\left\|_{\infty} \leqslant\right\| \bar{P}-P\left\|_{\infty}+\right\| P-y \|_{\infty}$, and a theorem on the interpolation error and its corollaries. For our purpose we use [12, Example 8.12]. By $P$ we designate a piecewise polynomial obtained in the same way like $\bar{P}$, but $P$ passes trough the points with the coordinates $\left(x_{i-1}, y\left(x_{i-1}\right)\right),\left(x_{i}, y\left(x_{i}\right)\right), i=1,2, \ldots, N$; instead of $\left(x_{i-1}, \bar{y}_{i-1}\right),\left(x_{i}, \bar{y}_{i+1}\right), i=1,2, \ldots, N$,

$$
P(x)=\left\{\begin{array}{cl}
p_{1}(x), & x \in\left[x_{0}, x_{1}\right], \\
p_{2}(x), & x \in\left[x_{1}, x_{2}\right], \\
\vdots & \\
p_{i}(x), & x \in\left[x_{i-1}, x_{i}\right], \\
\vdots & \\
p_{N}(x), & x \in\left[x_{N-1}, x_{N}\right],
\end{array}\right.
$$

where

$$
p_{i}(x)=\left\{\begin{array}{cc}
\frac{y_{i}-y_{i-1}}{x_{i}-x_{i-1}}\left(x-x_{i-1}\right)+y_{i-1}, & x \in\left[x_{i-1}, x_{i}\right], \\
0, & x \notin\left[x_{i-1}, x_{i}\right],
\end{array}\right.
$$

and $i=1,2, \ldots, N$. 
Taking into account the constructions $(6.1),(6.4)$ and Theorem 3 the following is true

$$
\|P-\bar{P}\|_{\infty} \leqslant\left(C \ln ^{2} N\right) / N^{2}, \quad x \in[0,1] .
$$

The first part is on the subinterval $[0, \lambda]$, this one corresponds with the mesh when $i=1,2, \ldots, N / 4$. Here, the mesh is equidistant i.e. $h_{i-1}=h_{i}$, and $h_{i}=$ $\mathcal{O}(\varepsilon \ln N / N)$. Using Theorem 1] [12, Example 8.12], $h_{i}=\mathcal{O}(\varepsilon \ln N / N)$ we have that

$$
\begin{aligned}
&\left|y(x)-p_{i}(x)\right| \leqslant \frac{h_{i}^{2}}{8} \max _{\xi \in\left[x_{i-1}, x_{i}\right]}\left|y^{\prime \prime}(\xi)\right| \leqslant C_{1} \frac{\varepsilon^{2} \ln ^{2} N}{N^{2}} \max _{\xi \in\left[x_{i-1}, x_{i}\right]}\left|s^{\prime \prime}(\xi)+r^{\prime \prime}(\xi)\right| \\
& \leqslant C_{2} \frac{\varepsilon^{2} \ln ^{2} N}{N^{2}} \max _{\xi \in\left[x_{i-1}, x_{i}\right]}\left|\varepsilon^{-2}\left(e^{-\frac{\xi}{\varepsilon} \sqrt{m}}+e^{-\frac{(\xi-1)}{\varepsilon} \sqrt{m}}\right)+r^{\prime \prime}(\xi)\right| \\
& \leqslant C_{2} \frac{\varepsilon^{2} \ln ^{2} N}{N^{2}}\left(\varepsilon^{-2}+C_{3}\right) \leqslant \frac{C \ln ^{2} N}{N^{2}}, i=1,2, \ldots, N / 4 .
\end{aligned}
$$

The remaining part of the proof, i.e. for $x \in\left[\lambda, x_{N / 4+1}\right] \cup\left[x_{N / 4+1}, 1 / 2\right]$ which corresponds with the mesh for $i=N / 4, N / 4+1, \ldots, N / 2$, is constructed as in [7].

For $i=N / 4+1, \ldots, N / 2$, the mesh isn't equidistant but holds $h_{i}=\mathcal{O}(1 / N)$. According to the Theorem 1 , to the Theorem 3 [12, Example] and the features of the mesh we obtain

$$
\left|y(x)-p_{i}(x)\right| \leqslant \frac{h_{i}^{2}}{8} \max _{\xi \in\left[x_{N / 4+1}, 1 / 2\right]}\left|y^{\prime \prime}(\xi)\right| \leqslant \frac{C}{N^{2}} .
$$

On $\left[\lambda, x_{N / 4}+1\right]$, according to the Theorem 1 we obtain

$$
\begin{aligned}
y-p_{i}(x) & =y-\frac{y_{i}-y_{i-1}}{x_{i}-x_{i-1}}\left(x-x_{i-1}\right)+y_{i-1} \\
& =s-\frac{s_{i}-s_{i-1}}{x_{i}-x_{i-1}}\left(x-x_{i-1}\right)+s_{i-1}+r-\frac{r_{i}-r_{i-1}}{x_{i}-x_{i-1}}\left(x-x_{i-1}\right)+r_{i-1} .
\end{aligned}
$$

For the layer component $s$, based on the estimate 2.2 , we have

$$
\begin{aligned}
\left|s-\frac{s_{i}-s_{i-1}}{x_{i}-x_{i-1}}\left(x-x_{i-1}\right)+s_{i-1}\right| & \leqslant|s|+\left|s_{i+1}-s_{i}\right|+\left|s_{i}\right| \\
& \leqslant C_{1}\left(e^{-\frac{x_{i-1}}{\varepsilon} \sqrt{m}}+e^{-\frac{x_{i-1}-1}{\varepsilon} \sqrt{m}}\right) \leqslant \frac{C}{N^{2}} .
\end{aligned}
$$

For the regular component $r$, we apply again the estimate from [12, Example 8.12], the estimate 2.1), and we obtain

$$
\left|r-\frac{r_{i}-r_{i-1}}{x_{i}-x_{i-1}}\left(x-x_{i-1}\right)+r_{i-1}\right| \leqslant \frac{h_{i-1}^{2}}{8} \max _{\xi \in\left[x_{i-1}, x_{i}\right]}\left|r^{\prime \prime}(\xi)\right| \leqslant \frac{C}{N^{2}} .
$$

Now, collecting (6.6), (6.7), (6.8), (6.9) and (6.10), the statement of the theorem is therefore proven. 
Cubic spline. In the numerical experiments we will use a natural cubic spline as a global solution. We construct it in the way as follows: design the natural cubic spline by $C$,

$$
C(x)=C_{i}(x), x \in\left[x_{i}, x_{i+1}\right], i=0,1, \ldots, N-1,
$$

where $C_{i}$ are the cubic functions

$$
\begin{aligned}
C_{i}(x)=M_{i} & \frac{\left(x_{i+1}-x\right)^{3}}{6 h_{i+1}}+M_{i+1} \frac{\left(x-x_{i}\right)^{3}}{6 h_{i+1}} \\
& +\left[\frac{\bar{y}_{i+1}-\bar{y}_{i}}{h_{i+1}}-\frac{h_{i+1}}{6}\left(M_{i+1}-M_{i}\right)\right]\left(x-x_{i}\right)+\bar{y}_{i}-M_{i} \frac{h_{i+1}^{2}}{6},
\end{aligned}
$$

the moments $M_{i}:=C_{i}^{\prime \prime}\left(x_{i}\right), i=1, N-1$ are obtained from the system

$$
\frac{h_{i}}{6} M_{i-1}+\frac{h_{i}+h_{i+1}}{3} M_{i}+\frac{h_{i+1}}{6} M_{i+1}=\frac{\bar{y}_{i+1}-\bar{y}_{i}}{h_{i+1}}-\frac{\bar{y}_{i}-\bar{y}_{i-1}}{h_{i}}, i=1, \ldots, N-1,
$$

and $M_{0}:=C_{0}^{\prime \prime}\left(x_{0}\right)=0, M_{N}:=C_{N-1}^{\prime \prime}\left(x_{N}\right)=0$.

\section{Numerical EXPERIMENTS}

In this section we conduct numerical experiments in order to confirm the theoretical results, i.e. to confirm the accuracy of the different scheme (3.5) on the meshes 2.7), 2.4, 2.8 and 2.9.

Example 1: We consider the following boundary value problem

$$
\begin{gathered}
\varepsilon^{2} y^{\prime \prime}=y+\cos ^{2} \pi x+2 \varepsilon^{2} \pi^{2} \cos ^{2} \pi x \text { on }(0,1), \\
y(0)=y(1)=0 .
\end{gathered}
$$

The exact solution of this problem is

$$
y(x)=\frac{e^{-\frac{x}{\varepsilon}}+e^{\frac{-(1-x)}{\varepsilon}}}{1+e^{-\frac{1}{\varepsilon}}}-\cos ^{2} \pi x .
$$

The nonlinear system was solved using the initial condition $y_{0}=-0.5$ and the value of the constant $\gamma=1$. Because of the fact that the exact solution is known, we compute the error $E_{N}$ and the rate of convergence Ord in the usual way

$$
\begin{gathered}
E_{N}=\left\|y-\bar{y}^{N}\right\|_{\infty}, \text { Ord }=\frac{\ln E_{N}-\ln E_{2 N}}{\ln (2 k /(k+1))}, \text { (Shishkin), } \\
\text { Ord }=\frac{\ln E_{N}-\ln E_{2 N}}{\ln 2}, \text { (Bakhvalov, Liseikin) }
\end{gathered}
$$

where $N=2^{k}, k=4,5, \ldots, 12$, and $y$ is the exact solution of the problem $1.1 \mathrm{a}$ (1), while $\bar{y}^{N}$ is an appropriate numerical solution of (4.1). The graphics of the numerical and exact solutions, for various values of the parameter $\varepsilon$ are on Figure 1 (left), while fragments of these solutions are on Figure 1 (right). The values of $E_{N}$ and Ord are in Tables 1. The graphics of the exact and global solution obtained by using a linear spline, and the corresponding error are shown on Figure 2 , while the graphics of the exact and global solution obtained by using a natural cubic spline, and the corresponding error are shown on Figure 3. 

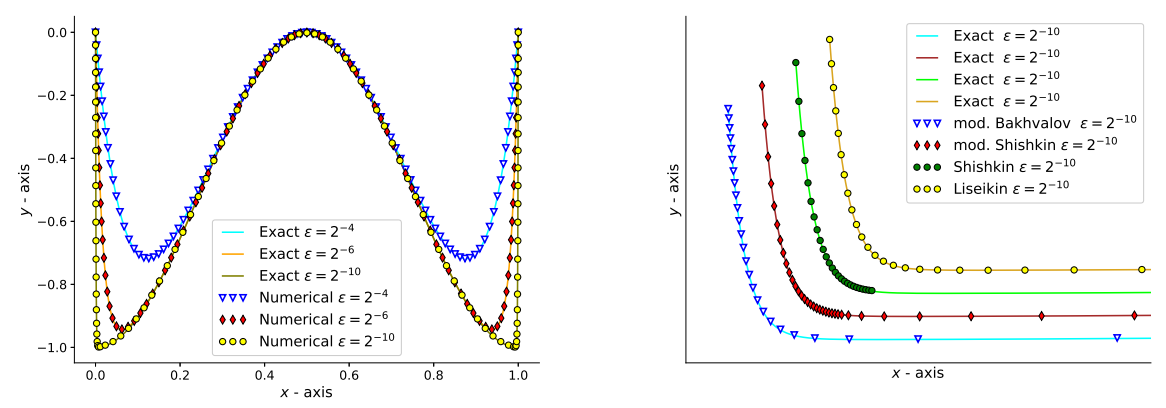

Figure 1. Exact and numerical solutions (left), layer near $x=0$ (right)
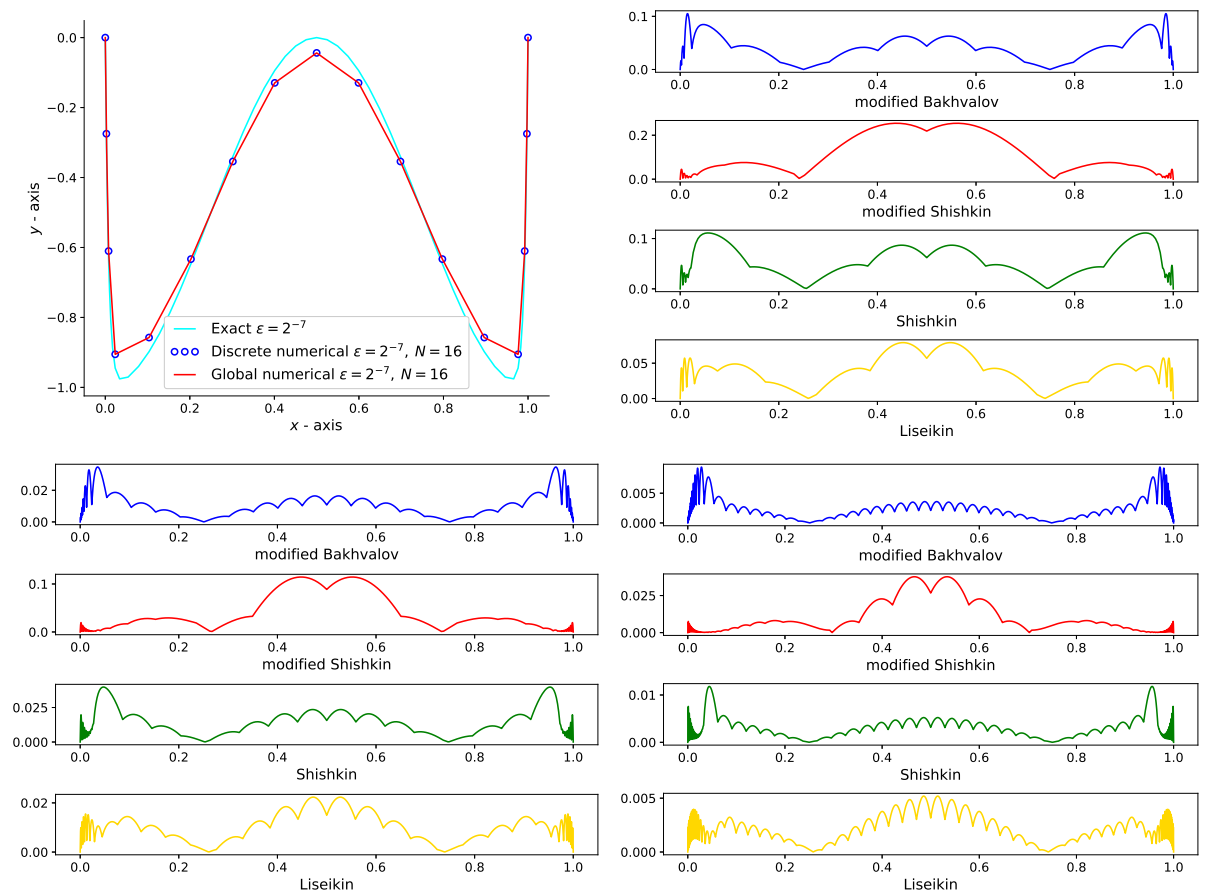

Figure 2. Exact, discrete and global numerical solutions (left up), error (right up $-N=16$, left down $-N=32$, right down$N=64)$ 
ON CONSTRUCTION OF A GLOBAL NUMERICAL SOLUTION...

\begin{tabular}{|c|c|c|c|c|c|c|c|c|c|c|}
\hline \multirow[b]{2}{*}{$N$} & \multicolumn{2}{|c|}{$2^{-5}$} & \multicolumn{2}{|c|}{$2^{-10}$} & \multicolumn{2}{|c|}{$2^{-20}$} & \multicolumn{2}{|c|}{$2^{-30}$} & \multicolumn{2}{|c|}{$2^{-40}$} \\
\hline & $E_{n}$ & Ord & $E_{n}$ & Ord & $E_{n}$ & Ord & $E_{n}$ & Ord & & \\
\hline $2^{4}$ & $1.015 \mathrm{e}-1$ & 2.08 & $1.255 \mathrm{e}-1$ & 2.67 & $1.278 \mathrm{e}-1$ & 2.65 & $1.278 \mathrm{e}-1$ & 2.65 & $1.278 \mathrm{e}-1$ & 2.65 \\
\hline $2^{5}$ & $3.811 \mathrm{e}-2$ & 2.83 & $3.570 \mathrm{e}-2$ & 2.65 & $3.666 \mathrm{e}-2$ & 2.64 & $3.666 \mathrm{e}-2$ & 2.64 & $3.666 \mathrm{e}-2$ & 2.64 \\
\hline $2^{6}$ & $8.956 \mathrm{e}-3$ & 2.87 & $9.194 \mathrm{e}-3$ & 2.20 & $9.515 \mathrm{e}-3$ & 2.26 & $9.515 \mathrm{e}-3$ & 2.26 & $9.515 \mathrm{e}-3$ & 2.26 \\
\hline $2^{7}$ & $1.900 \mathrm{e}-3$ & 2.74 & $2.804 \mathrm{e}-3$ & 1.99 & $2.803 \mathrm{e}-3$ & 1.99 & $2.803 \mathrm{e}-3$ & 1.99 & $2.804 \mathrm{e}-3$ & 1.99 \\
\hline $2^{8}$ & $4.099 \mathrm{e}-4$ & 2.61 & $9.198 \mathrm{e}-4$ & 2.00 & $9.196 \mathrm{e}-4$ & 2.00 & $9.196 \mathrm{e}-4$ & 2.00 & $9.196 \mathrm{e}-4$ & 2.00 \\
\hline $2^{9}$ & $9.104 \mathrm{e}-5$ & 2.52 & $2.911 \mathrm{e}-4$ & 2.00 & $2.911 \mathrm{e}-4$ & 2.00 & $2.911 \mathrm{e}-4$ & 2.00 & $2.911 \mathrm{e}-4$ & 2.00 \\
\hline $2^{10}$ & $2.069 \mathrm{e}-5$ & 2.42 & $8.987 \mathrm{e}-5$ & 2.00 & $8.986 e-5$ & 2.00 & $8.986 \mathrm{e}-5$ & 2.00 & $8.986 \mathrm{e}-5$ & 2.00 \\
\hline $2^{11}$ & $4.851 \mathrm{e}-6$ & 2.33 & $2.719 \mathrm{e}-5$ & 2.00 & $2.719 \mathrm{e}-5$ & 2.00 & $2.719 \mathrm{e}-5$ & 2.00 & $2.719 \mathrm{e}-5$ & 2.00 \\
\hline $2^{12}$ & $1.180 \mathrm{e}-6$ & - & $8.091 \mathrm{e}-6$ & - & $8.090 \mathrm{e}-6$ & - & $8.090 \mathrm{e}-6$ & - & $8.090 \mathrm{e}-6$ & - \\
\hline \multicolumn{11}{|c|}{ mesh 2.7} \\
\hline $2^{4}$ & $1.944 \mathrm{e}-1$ & 2.29 & $2.356 \mathrm{e}-1$ & 1.80 & $2.408 \mathrm{e}-1$ & 1.76 & $2.408 \mathrm{e}-1$ & 1.766 & $2.408 \mathrm{e}-1$ & 1.76 \\
\hline $2^{5}$ & $6.598 \mathrm{e}-2$ & 3.06 & $1.010 \mathrm{e}-1$ & 2.20 & $1.049 \mathrm{e}-1$ & 2.17 & $1.050 \mathrm{e}-1$ & 2.17 & $1.050 \mathrm{e}-1$ & 2.17 \\
\hline $2^{6}$ & $1.377 \mathrm{e}-2$ & 3.13 & $3.276 \mathrm{e}-2$ & 2.36 & $3.450 \mathrm{e}-2$ & 2.34 & $3.450 \mathrm{e}-2$ & 2.34 & $3.450 \mathrm{e}-2$ & 2.34 \\
\hline $2^{7}$ & $2.547 \mathrm{e}-3$ & 2.76 & $9.157 \mathrm{e}-3$ & 2.40 & $9.768 \mathrm{e}-3$ & 2.37 & $9.769 \mathrm{e}-3$ & 2.37 & $9.769 \mathrm{e}-3$ & 2.37 \\
\hline $2^{8}$ & $5.413 \mathrm{e}-4$ & 2.57 & $2.381 \mathrm{e}-3$ & 2.42 & $2.581 \mathrm{e}-3$ & 2.36 & $2.581 \mathrm{e}-3$ & 2.36 & $2.581 \mathrm{e}-3$ & 2.36 \\
\hline $2^{9}$ & $1.226 \mathrm{e}-4$ & 2.50 & $5.907 \mathrm{e}-4$ & 2.51 & $6.619 \mathrm{e}-4$ & 2.33 & $6.620 \mathrm{e}-4$ & 2.33 & $6.620 \mathrm{e}-4$ & 2.33 \\
\hline $2^{10}$ & $2.818 \mathrm{e}-5$ & 2.45 & $1.343 \mathrm{e}-4$ & 2.82 & $1.674 \mathrm{e}-4$ & 2.30 & $1.674 \mathrm{e}-4$ & 2.30 & $1.674 \mathrm{e}-4$ & 2.30 \\
\hline $2^{11}$ & $6.478 \mathrm{e}-6$ & 2.43 & $2.487 \mathrm{e}-5$ & 2.92 & $4.211 \mathrm{e}-5$ & 2.28 & $4.211 \mathrm{e}-5$ & 2.28 & $4.212 \mathrm{e}-5$ & 2.28 \\
\hline $2^{12}$ & $1.484 \mathrm{e}-6$ & - & $4.233 \mathrm{e}-6$ & - & $1.055 \mathrm{e}-5$ & - & $1.055 \mathrm{e}-5$ & - & $1.055 \mathrm{e}-5$ & - \\
\hline \multicolumn{11}{|c|}{ mesh 2.4} \\
\hline $2^{4}$ & $3.038 \mathrm{e}-2$ & 1.97 & $5.847 \mathrm{e}-2$ & 1.89 & $6.790 \mathrm{e}-2$ & 1.87 & $6.822 \mathrm{e}-2$ & 1.86 & $6.823 \mathrm{e}-2$ & 1.86 \\
\hline $2^{5}$ & $7.750 \mathrm{e}-3$ & 1.94 & $1.577 \mathrm{e}-2$ & 1.98 & $1.857 \mathrm{e}-2$ & 1.97 & $1.867 \mathrm{e}-2$ & 1.97 & $1.867 \mathrm{e}-2$ & 1.96 \\
\hline $2^{6}$ & $2.017 \mathrm{e}-3$ & 1.96 & $4.009 \mathrm{e}-3$ & 1.89 & $4.754 \mathrm{e}-3$ & 1.99 & $4.779 \mathrm{e}-3$ & 1.99 & $4.780 \mathrm{e}-3$ & 1.99 \\
\hline $2^{7}$ & $5.163 e-4$ & 1.99 & $1.076 \mathrm{e}-3$ & 1.68 & $1.195 \mathrm{e}-3$ & 2.00 & $1.202 \mathrm{e}-3$ & 2.00 & $1.202 \mathrm{e}-3$ & 2.00 \\
\hline $2^{8}$ & $1.295 \mathrm{e}-4$ & 2.00 & $3.355 \mathrm{e}-4$ & 2.08 & $2.993 \mathrm{e}-4$ & 2.00 & $3.009 \mathrm{e}-4$ & 2.00 & $3.010 \mathrm{e}-4$ & 2.00 \\
\hline $2^{9}$ & $3.246 e-5$ & 2.00 & $7.912 \mathrm{e}-5$ & 2.54 & $7.487 \mathrm{e}-5$ & 2.00 & $7.527 \mathrm{e}-5$ & 2.00 & $7.528 \mathrm{e}-5$ & 2.00 \\
\hline $2^{10}$ & $8.117 \mathrm{e}-6$ & 2.00 & $1.357 \mathrm{e}-5$ & 2.00 & $1.872 \mathrm{e}-5$ & 1.99 & $1.882 \mathrm{e}-5$ & 2.00 & $1.882 \mathrm{e}-5$ & 2.00 \\
\hline $2^{11}$ & $2.029 \mathrm{e}-6$ & 2.00 & $3.397 \mathrm{e}-6$ & 2.00 & $4.704 \mathrm{e}-6$ & 1.85 & $4.705 \mathrm{e}-6$ & 2.00 & $4.706 \mathrm{e}-6$ & 2.00 \\
\hline $2^{12}$ & $5.073 \mathrm{e}-7$ & - & $8.494 \mathrm{e}-7$ & - & $1.300 \mathrm{e}-6$ & - & $1.176 \mathrm{e}-6$ & - & $1.176 \mathrm{e}-6$ & - \\
\hline \multicolumn{11}{|c|}{ mesh 2.8} \\
\hline $2^{4}$ & $1.209 \mathrm{e}-2$ & 2.43 & $3.055 \mathrm{e}-2$ & 1.96 & $3.593 \mathrm{e}-2$ & 1.95 & $3.654 \mathrm{e}-2$ & 1.94 & $3.660 \mathrm{e}-2$ & 1.94 \\
\hline $2^{5}$ & $2.234 \mathrm{e}-3$ & 2.18 & $7.873 \mathrm{e}-3$ & 1.69 & $9.332 \mathrm{e}-3$ & 1.97 & $9.496 \mathrm{e}-3$ & 1.85 & $9.513 \mathrm{e}-3$ & 1.97 \\
\hline $2^{6}$ & $4.897 \mathrm{e}-4$ & 2.05 & $2.444 \mathrm{e}-3$ & 1.78 & $2.355 \mathrm{e}-3$ & 2.00 & $2.397 \mathrm{e}-2$ & 2.00 & $2.401 \mathrm{e}-3$ & 2.00 \\
\hline $2^{7}$ & $1.177 \mathrm{e}-4$ & 2.01 & $7.102 \mathrm{e}-4$ & 1.96 & $5.902 \mathrm{e}-4$ & 2.00 & $6.000 \mathrm{e}-4$ & 2.00 & $6.017 \mathrm{e}-4$ & 2.00 \\
\hline $2^{8}$ & $2.913 e-5$ & 2.00 & $1.819 \mathrm{e}-4$ & 2.48 & $1.476 \mathrm{e}-4$ & 2.00 & $1.502 \mathrm{e}-4$ & 2.00 & $1.505 \mathrm{e}-4$ & 2.00 \\
\hline $2^{9}$ & $7.264 \mathrm{e}-6$ & 2.00 & $3.255 \mathrm{e}-5$ & 3.27 & $4.469 e-5$ & 2.00 & $3.757 \mathrm{e}-5$ & 2.00 & $3.764 \mathrm{e}-5$ & 2.00 \\
\hline $2^{10}$ & $1.814 \mathrm{e}-6$ & 2.00 & $3.355 \mathrm{e}-6$ & 1.99 & $1.354 \mathrm{e}-5$ & 2.00 & $9.393 e-6$ & 2.00 & $9.410 \mathrm{e}-6$ & 2.00 \\
\hline $2^{11}$ & $4.536 e-7$ & 2.00 & $8.462 \mathrm{e}-7$ & 1.54 & $3.867 \mathrm{e}-6$ & 2.00 & $2.348 \mathrm{e}-6$ & 2.00 & $2.352 \mathrm{e}-6$ & 2.00 \\
\hline $2^{12}$ & $1.134 \mathrm{e}-7$ & - & $2.905 e-7$ & - & $1.196 \mathrm{e}-6$ & - & $6.604 \mathrm{e}-7$ & - & $5.881 \mathrm{e}-7$ & - \\
\hline
\end{tabular}

TABLE 1. Values of $E_{N}$ and Ord 

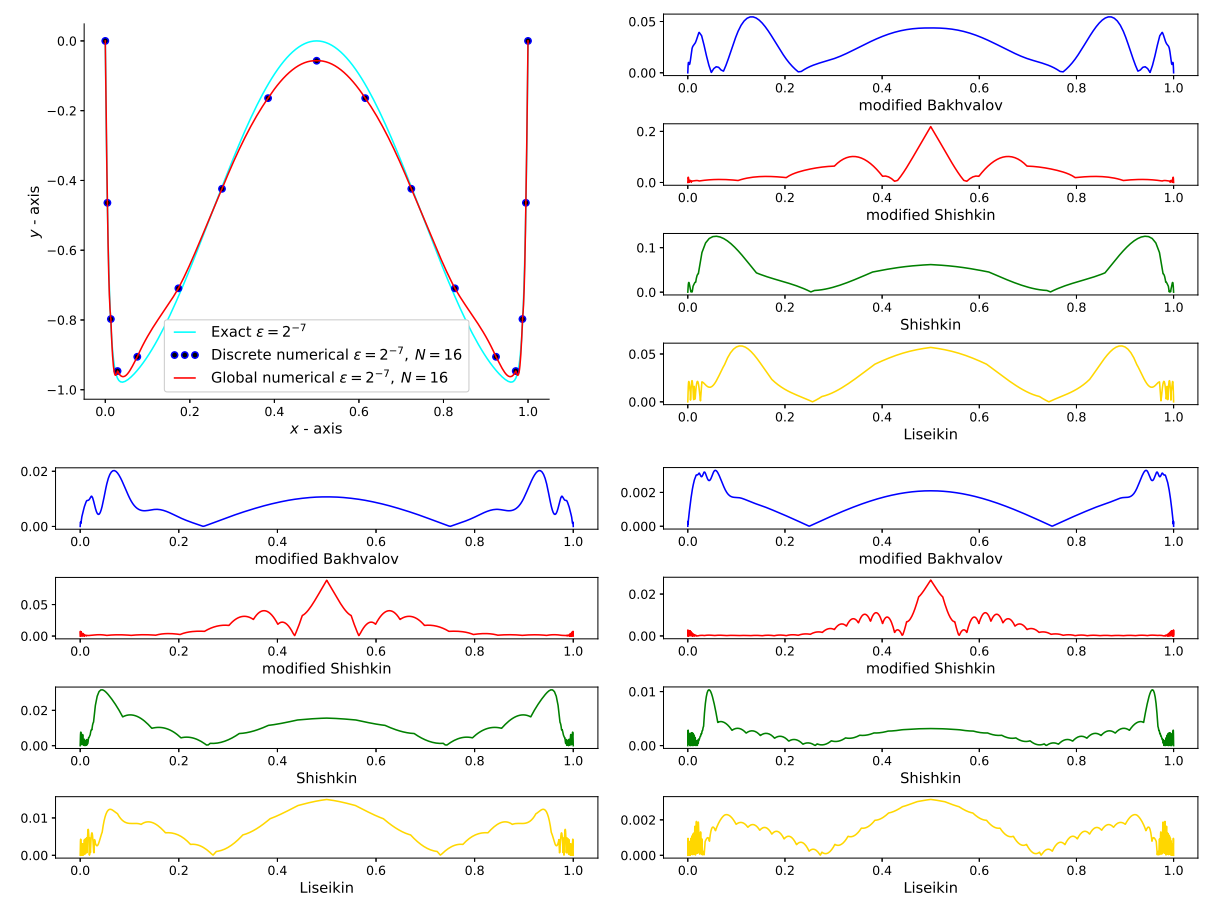

Figure 3. Exact, discrete and global numerical solutions (left up), error (right up $-N=16$, left down $-N=32$, right down$N=64)$

\section{Conclusion}

In the present paper we constructed a numerical solution for the one-dimensional singularly-perturbed reaction-diffusion boundary-value problem. The class of different schemes was constructed, and we proved the existence and uniqueness of the discrete numerical solution. After that, we proved $\varepsilon$-uniformly convergence of the constructed class of different schemes on the modified Shishkin mesh of order 2. A global numerical solution was constructed based on a linear spline and proved that the order of the error value is $\mathcal{O}\left(\ln ^{2} N / N^{2}\right)$. The numerical experiments at the end of the paper confirm the theoretical results. The results obtained by using a global numerical solution based on a natural cubic spline and the Shishkin, the modified Bakhvalov and last but not least the Liseikin mesh are included in the numerical experiments. Although, the theoretical analysis for these meshes wasn't done, the results suggest that the order of convergence is 2 for all of them. Especially, good results have been achieved by using the Liseikin mesh. 
Acknowledgement. Thanks are due to the referee for her/his hard work on the review process.

\section{REFERENCES}

[1] I. P. Boglaev, Approximate solution of a non-linear boundary value problem with a small parameter for the highest-order differential, Zh. Vychisl. Mat. Mat. Fiz., 24(11), (1984), $1649-1656$.

[2] D. Herceg, Uniform fourth order difference scheme for a singular perturbation problem, Numer. Math., 56(7), (1989), 675-693.

[3] D. Herceg, K. Surla, Solving a nonlocal singularly perturbed problem by spline in tension, Novi Sad J. Math, 21(2), (1991), 119-132.

[4] D. Herceg, M. Miloradović, On numerical solution of semilinear singular perturbation problems by using the Hermite scheme on a new Bakhvalov-type mesh, Novi Sad J. Math, 33(1), (2003), 145-162.

[5] D. Herceg, Du. Herceg, On a fourth-order finite difference method for nonlinear twopoint boundary value problems, Novi Sad J. Math, 33(2), (2003), 173-180.

[6] S. Karasuljić, E. Duvnjaković, H. Zarin, Uniformly convergent difference scheme for a semilinear reaction-diffusion problem, Adv. Math. Sci. J., 4(2), (2015), 139-159.

[7] S. Karasuljić, E. Duvnjaković, V. Pašić, E. Baraković, Construction of a global solution for the one dimensional singularly-perturbed boundary value problem, Journal of Modern Methods in Numerical Mathematics, 8(1-2), (2017), 52-65.

[8] N. Kopteva, T. Linss, Uniform second-order pointwise convergence of a central difference approximation for a quasilinear convection-diffusion problem, J. Comput. Appl. Math., 137(2), (2001), 257-267.

[9] N. Kopteva, M. Stynes, A robust adaptive method for a quasi-linear one-dimensional convection-diffusion problem, SIAM J. Numer. Anal., 39(4), (2001), 1446-1467.

[10] N. Kopteva, M. Stynes, Numerical analysis of a singularly perturbed nonlinear reactiondiffusion problem with multiple solutions, Appl Numer Math, 51(2), (2004), 273-288.

[11] N. Kopteva, M. Pickett, H. Purtill, A robust overlapping Schwarz method for a singularly perturbed semilinear reaction-diffusion problem with multiple solutions. Int J Numer Anal Model, 6, (2009), 680-695.

[12] R. Kress, Numerical analysis, Springer-Verlag, New York, USA, 1998.

[13] T. Linss, H. G. Roos, R. Vulanović, Uniform pointwise convergence on Shishkin-type meshes for quasi-linear convection-diffusion problems. SIAM J Numer Anal, 38(3), (2000), $897-912$.

[14] T. Linss, G. Radojev, H. Zarin, Approximation of singularly perturbed reaction-diffusion problems by quadratic $C^{1}$-splines. Numer Algorithms, 61(1), (2012), 35-55.

[15] V.D. Liseikin, Grid Generation for Problems with Boundary and Interior Layers, Novosibirsk State University, Novosibirsk, Russia, 2018.

[16] V.D. Liseikin, V.I. Poansonen, Compact Difference Schemes and Layer Resolving Grids for Numerical Modeling of Problems with Boundary and Interior Layers, Numer. Analys. Appl., 12, (2019), 37-50.

[17] V.D. Liseikin, S. Karasuluic, Numerical analysis of grid-clustering rules for problems with power of the first type boundary layers, Computational technologies, 25(1), (2020), 49-66.

[18] J. Lorenz, Stability and monotonicity properties of stiff quasilinear boundary problems, Novi Sad J. Math, 12, (1982), 151-176.

[19] J. M. Ortega, W.C. Rheinboldt, Iterative Solution of Nonlinear Equations in Several Variables, Philadelphia, PA, USA, SIAM, 2000.

[20] G. I. Shishin, Grid approximation of singularly perturbed parabolic equations with internal layers, Sov. J. Numer. Anal. M. Russ. J. Numer. Anal. Math. Model, 3(5), (1988), 393-408. 
[21] G. Sun, M. Stynes, A uniformly convergent method for a singularly perturbed semilinear reaction-diffusion problem with multiple solutions, Math. Comp., 65(215), (1996), 10851109.

[22] M. Stynes, N. Kopteva, Numerical analysis of singularly perturbed nonlinear reactiondiffusion problems with multiple solutions, Comput. Math. Appl., 51(5), (2006), 857-864.

[23] K. Surla, Z. Uzelac, On Stability of Spline Difference Scheme for Reaction-Diffusion Time-Dependent Singularly Perturbed Problem, Novi Sad J. Math., 33(2), (2003), 89-94.

[24] R. Vulanović, On a numerical solution of a type of singularly perturbed boundary value problem by using a special discretization mesh, Novi Sad J. Math., 13, (1983), 187-201.

[25] R. Vulanović, Mesh generation methods for numerical solution of quasilinear singular perturbation problems, Novi Sad J. Math., 19(2), (1989), 171-193.

[26] R. Vulanović, A second order numerical method for non-linear singular perturbation problems without turning points, USSR Comp. Math., 31(4), (1991), 522-532.

[27] R. Vulanović, On numerical solution of semilinear singular perturbation problems by using the Hermite scheme, Novi Sad J. Math., 23(2), (1993), 363-379.

[28] R. Vulanović, A Higher-order Scheme for Quasilinear Boundary Value Problems with Two Small Parameters, Computing, 67(4), (2001), 287-303.

[29] R. Vulanović, An almost sixth-order finite-difference method for semilinear singular perturbation problems, Comput. Methods Appl. Math., 4(3), (2004), 368-383.

Šabana Zahirovića 6,75000 TuZla

UNIVERSITY OF TUZLA,

Faculty of Sciences and Mathematics,

Univerzitetska 4,75000 TuZla

Bosnia and Herzegovina

Email address: samir.karasuljic@untz.ba

Trepče 6, TešanJ

UNiversity of TUZLA,

Faculty of Sciences and Mathematics,

Univerzitetska 4, 75000 Tuzla,

Bosnia and Herzegovina

Email address: hidajeta1993@gmail.com

Received 16.9.2020

Revised 28.12.2020

Accepted 31.12.2020 